\title{
Semi-conducteurs magnétiques dilués
}

\author{
Lucien Besombes ${ }^{1}$, David Ferrand ${ }^{1}$, Henri Mariette ${ }^{1}$ et Joël Cibert ${ }^{2}$ \\ 1. Équipe CEA-CNRS-UJF Nanophysique et Semi-conducteurs, Laboratoire de Spectrométrie Physique, \\ St Martin d'Hères, et Département de Recherche Fondamentale sur la Matière Condensée, CEA-Grenoble \\ 2. Laboratoire Louis Néel, CNRS, Grenoble
}

\begin{abstract}
En introduisant des impuretés magnétiques dans les semi-conducteurs, on obtient des matériaux dont les propriétés électroniques et magnétiques sont fortement couplées. On peut alors utiliser les spécificités des semi-conducteurs et les techniques de la microélectronique pour contrôler une aimantation par un champ électrique ou isoler un spin unique dans une boîte quantique adressable ; inversement, on peut agir sur le spin des porteurs de charge et réaliser ainsi certaines fonctions requises par l'électronique de spin.
\end{abstract}

\section{Introduction}

L'électronique repose sur la manipulation de porteurs de charges électriques dans des assemblages permettant de leur appliquer des champs électriques. À partir du milieu du XX siècle, les triodes et autres pentodes ont été remplacées par des composants solides, utilisant des matériaux bien choisis : les semi-conducteurs. En effet, dans les métaux, des électrons « libres » (susceptibles de se déplacer sous l'effet d'un champ électrique) en forte densité apportent une bonne conductivité mais écrantent tout champ électrique appliqué. Dans les isolants, au contraire, il est très facile d'établir un champ électrique mais très difficile de faire passer un courant, car la bande de conduction est vide. Les semi-conducteurs sont intermédiaires : ce sont des isolants tels qu'on peut introduire des électrons dans la bande de conduction (ou des trous dans la bande de valence), en densité suffisante pour leur conférer une conductivité raisonnable, mais suffisamment faible pour qu'ils n'écrantent pas irrémédiablement tout champ électrique appliqué. C'est ce qu'on appelle le dopage du semi-conducteur : on utilise pour cela des impuretés qui apportent plus ou moins d'électrons que les atomes qu'ils remplacent. Ces matériaux ont eu un début difficile. En 1931, Pauli écrivait : "One shouldn't work with semiconductors, that is a filthy mess; who knows whether they really exist?" C'est bien sûr le mauvais contrôle du taux d'impuretés qui rendait Pauli si pessimiste. Depuis, l'industrie de l'électronique a appris à maîtriser la pureté et la qualité cristalline de semiconducteurs comme le silicium (Si) ou l'arséniure de gallium (GaAs). II n'y a plus qu'à combiner faible densité de porteurs d'une part, application de champs électriques d'autre part, et on a un transistor à effet de champ, où la tension de grille module la conductivité entre source et drain. Et il y a un plus : dans un semi-conducteur comme GaAs, on peut aussi obtenir des porteurs libres en illuminant le semi-conducteur; un électron de la bande de valence acquiert l'énergie suffisante pour passer dans la bande de conduction en absorbant un photon. Comme le processus inverse, l'émission d'un photon, est aussi possible, c'est toute l'opto-électronique qui suit...

Plus récemment, on a inventé les hétérostructures de semiconducteurs : pour cela, on associe, dans un même cristal, deux matériaux différents ; par exemple, un semi-conducteur et un isolant, comme le silicium $\mathrm{Si}$ et son oxyde $\mathrm{SiO}_{2}$. Les électrons restent alors confinés dans le semi-conducteur et peuvent s'y déplacer sous l'effet de champs électriques. Les mêmes effets de confinement sont réalisés avec un couple associant un semi-conducteur de petite bande interdite et un autre de grande bande interdite (appelé " barrière ») : les couples Ge et Si d'une part, GaAs et AlAs d'autre part, sont les plus utilisés. Cela permet d'inclure dans le matériau des structures complexes, beaucoup plus complexes que ce que l'on pourrait réaliser en appliquant des champs électriques par des contacts, avec des dimensions caractéristiques qui sont devenues de plus en plus petites au cours des années. De l'ordre de la dizaine de microns dans les " circuits inté- grés " des années 80 , elles atteignent maintenant quelques nanomètres dans ce qu'on appelle des nanostructures. Ce principe général - des paquets d'électrons se déplaçant entre des barrières sous l'effet de champs électriques - a permis l'essor de la microélectronique et de l'informatique, en rendant possibles tous les composants aptes au traitement de l'information : amplificateurs, portes logiques, processeurs, mémoires flash, et les RAM (Random Access Memory), ces mémoires à accès rapide, mais volatiles, dont on rajoute parfois une barrette dans son micro-ordinateur.

Car il ne suffit pas de savoir traiter l'information, il faut aussi l'enregistrer. Le stockage de masse repose sur des principes et des matériaux complètement différents : l'aimantation rémanente dans les matériaux magnétiques. Tout vient encore des électrons, qui, outre leur charge électrique, portent un spin (un moment cinétique élémentaire) auquel est associé un moment magnétique. Celui-ci s'oriente sous l'effet d'un champ magnétique, mais l'énergie mise en jeu est très faible : dans un champ, déjà élevé, de 1 tesla, cette énergie Zeeman est 200 fois plus faible que l'énergie thermique à température ambiante. Pas question de confier la moindre information à un objet aussi peu stable. Tout change grâce aux interactions entre spins, surtout lorsque cette interaction aligne les spins entre eux dans un matériau ferromagnétique. Des domaines magnétiques se forment alors, qui interagissent entre eux et avec les défauts du matériau. La configuration est tellement stable que des domaines alignés par un champ magnétique ne reprennent pas une orientation aléatoire lorsqu'on coupe le champ : il reste une aimantation rémanente, que l'on ne peut retourner qu'en appliquant un champ de sens opposé, le champ coercitif. Le champ magnétique d'une tête d'enregistrement écrit l'information sur la bande magnétique ou le disque dur, sous forme d'une aimantation rémanente locale, qui peut être lue plusieurs fois et ne demande pas d'énergie pour être maintenue. Mais il faut pour cela positionner la tête de lecture au bon endroit : un disque dur permet de stocker beaucoup d'informations de façon non volatile, mais l'accès est lent.

On rêve d'associer les deux techniques: manipulation de charge et manipulation de l'aimantation. C'est ce qu'on appelle l'électronique de spin, qui vise non seulement à un stockage de l'information plus efficace, mais aussi à utiliser l'aimantation et le spin dans le traitement de l'information, dans des applications qui vont de la logique reprogrammable à l'information quantique.

Quels matériaux doit-on utiliser pour cela ? Les premières applications de l'électronique de spin juxtaposent des métaux magnétiques et des composants à semi-conducteurs. Les têtes de lecture compactes et sensibles qui équipent désormais les lecteurs de disques durs exploitent des multicouches de métaux magnétiques, dont la conductivité varie très fortement sous l'effet d'un champ magnétique. Dans les mémoires MRAM (Magnetic RAM) qui arrivent sur le marché et qui combinent accès rapide et stockage de masse non volatile, cette magnéto-résistance géante est lue par des composants à 
semi-conducteurs qui assurent l'adressage et la mesure. La fabrication de structures hybrides, où matériaux magnétiques et semi-conducteurs sont imbriqués, réaliserait une intégration à plus petite échelle, et permettrait en outre d'utiliser comme support de l'information non seulement l'intensité du courant circulant dans le semi-conducteur, mais aussi sa polarisation en spin. Une intégration plus poussée encore consisterait à rendre le semi-conducteur lui-même magnétique : c'est ce que visent les semi-conducteurs magnétiques dilués, l'information étant alors portée par les porteurs (semiconducteurs ferromagnétiques) ou par les spins localisés (spin dans une boîte quantique).

Mais ce n'est pas tout. Tant que les composants de l'électronique manipulaient quelques millions d'électrons sur des dimensions micrométriques, on pouvait ignorer la mécanique quantique - ou plutôt, une fois la structure de bande connue, on pouvait utiliser des équations du mouvement ressemblant à s'y méprendre à celles de la mécanique classique. Pauli avait snobé les semi-conducteurs, les spécialistes des composants électroniques le lui rendaient bien! Mais les dimensions des composants électroniques atteignent maintenant quelques nanomètres : on sait confiner les électrons dans une couche très fine (un " puits quantique ») dans laquelle ils se déplaceront suivant seulement deux dimensions (figure 1b), ou dans un " fil quantique " où ils n'ont plus qu'un degré de liberté, ou enfin dans une "boîte quantique » (figure 1c). À cette échelle où on ne manipule plus qu'un petit nombre d'électrons, voire un seul, sur des dimensions nanométriques, les effets quantiques ne peuvent être ignorés. Ce qui apparaît comme un problème dans la "roadmap" qui prévoit l'évolution de la microélectronique, est vu comme une formidable ouverture par les promoteurs de l'information quantique. L'informatique actuelle - classique - utilise un mode de calcul binaire : un bit d'information vaut soit 0 , soit 1 . En termes d'enregistrement par exemple, l'aimantation pointe « vers le bas » ou pointe "vers le haut », toute autre possibilité étant soigneusement éliminée. En mécanique quantique, un électron peut prendre l'un des deux états correspondants, spin vers le bas, ou spin vers le haut. Mais il peut aussi prendre un état dit superposition de ces deux états : c'est beaucoup mieux qu'un état intermédiaire ; il s'agit en fait d'un état où les
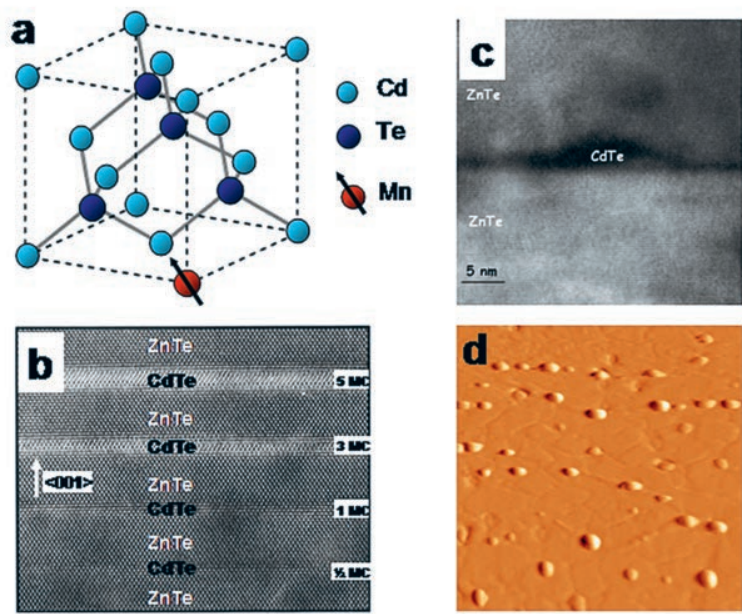

Figure 1. Épitaxie de semi-conducteurs. (a) Structure cristalline de CdTe (et $\mathrm{ZnTe}$ ), avec la substitution d'un atome de Cd par un atome de Mn qui rend le semi-conducteur magnétique. Suivant les conditions de croissance, on sait obtenir des couches d'épaisseur uniforme (b) formant des puits quantiques dont l'épaisseur est indiquée en monocouches (1 $M C=0,3 \mathrm{~nm}$ ), ou des inclusions d'un matériau dans l'autre (c) formant des boites quantiques. Sur les coupes observées en microscopie électronique à haute résolution $(b, c)$ on remarque la continuité de l'arrangement cristallin à travers les interfaces ("épitaxie "). L'image (d) est obtenue en microscopie à force atomique après le dépôt de CdTe sur ZnTe, avant de "refermer les boîtes » par le dernier dépôt de ZnTe. deux états extrêmes, 0 et 1 , sont réalisés en même temps. Pour l'information quantique, un tel système est un qu-bit. Un calcul quantique effectué sur un qu-bit remplace les calculs effectués pour toutes les valeurs possibles du bit classique. Pour un seul qu-bit, on peut générer une superposition des deux valeurs à la fois ; en généralisant pour $\mathrm{N}$ qu-bits, on pourrait préparer des superpositions de $2^{\mathrm{N}}$ valeurs à la fois. Imaginons la puissance de calcul d'un réseau de qu-bits à trois dimensions, de 10 qu-bits de côté... Reste à fabriquer ces qu-bits, et l'une des pistes est d'utiliser des impuretés magnétiques individuelles, adressables, dans un solide. On verra aussi ce qu'apportent à ce sujet les semi-conducteurs magnétiques dilués.

L'objectif de ces études est donc de partir de semi-conducteurs bien maîtrisés, qu'on sait doper électriquement, et qu'on sait associer dans des nanostructures : ce sont les ingrédients de la nanoélectronique; puis d'introduire d'autres impuretés, magnétiques. L'enjeu est alors de bien utiliser l'interaction qui apparaît entre les porteurs de charge électrique et les impuretés magnétiques, soit pour manipuler les propriétés magnétiques par l'intermédiaire des porteurs (grâce aux techniques de la microélectronique), soit pour contrôler non seulement la charge des porteurs, mais aussi leur spin.

\section{Semi-conducteurs magnétiques dilués non dopés : l'effet Zeeman géant}

Ces matériaux ont été inventés il y a environ 30 ans par les physiciens de Varsovie : en introduisant une impureté magnétique, le manganèse, dans les semi-conducteurs II-VI, des effets magnéto-optiques exceptionnels [1] ont été observés.

Les semi-conducteurs courants ont une structure cristalline proche de la structure diamant, formée de deux réseaux cubiques à faces centrées interpénétrés (figure 1a). Dans le silicium, les deux sous-réseaux sont constitués du même élément de la colonne IV du tableau de Mendeleïev. En formant un sous-réseau par un élément de la colonne III (comme le gallium $\mathrm{Ga}$ ) et l'autre par un élément de la colonne $\mathrm{V}$ (comme l'arsenic As), le nombre total d'électrons est le même, et les propriétés électroniques du semi-conducteur III-V (GaAs) restent analogues, avec des valeurs différentes de certains paramètres comme la largeur de la bande interdite. De la même façon, on obtient un semi-conducteur II-VI avec un sousréseau constitué d'éléments de la colonne II (le cadmium $\mathrm{Cd}$ ou le zinc $\mathrm{Zn}$ ) et l'autre constitué d'éléments de la colonne $\mathrm{VI}$ (comme le tellure Te).

Si l'on regarde alors la quatrième ligne du tableau périodique, la différence entre la configuration électronique des éléments $\mathrm{Zn}$ et $\mathrm{Mn}$ ne concerne pas les couches à partir desquelles se forment la bande de valence et la bande de conduction du semi-conducteur ZnTe : dans les deux éléments, la couche $4 s$ est pleine et la couche $4 p$ est vide. La différence porte seulement sur la couche $3 d$, relativement interne : elle est pleine (10 électrons) pour $\mathrm{Zn}$, il y a donc autant d'électrons de spins "up" que de spin "down" et pas de moment magnétique résultant ; elle est à moitié pleine dans $\mathrm{Mn}$, élément de transition, et la règle de Hund stipule que les 5 électrons s'organisent pour maximiser le moment magnétique. On s'attend donc à ce que l'impureté Mn en substitution de $\mathrm{Zn}$ ne change pas les propriétés électroniques du semi-conducteur mais apporte un spin $S=5 / 2$, localisé sur l'impureté.

Et c'est bien ce qui se passe, avec trois bonnes surprises d'abord, $\mathrm{Mn}$ prend la place de $\mathrm{Zn}$, de façon aléatoire, en toutes proportions de 0 à $100 \%$; ensuite, la qualité du matériau obtenu est excellente, en particulier sur le plan des propriétés optiques ; enfin, non seulement cela confère au semiconducteur un comportement paramagnétique, mais également il y a un fort couplage entre ces propriétés magnétiques et les propriétés optiques, ce qui apporte des propriétés magnéto-optiques exceptionnelles, vite qualifiées de géantes. 
Et ce qui est vrai pour (Zn, Mn)Te s'applique à toute la série des semi-conducteurs II-VI : (Cd, Mn)Te, (Zn, Mn)Se..

Le comportement paramagnétique vient simplement du comportement indépendant de chaque spin $S=5 / 2$. En présence d'un champ magnétique appliqué $\boldsymbol{H}$, la dégénérescence $(2 S+1)=6$ est levée par l'effet Zeeman et la population des six niveaux est régie par la distribution de Boltzmann : un calcul direct montre que, pour un champ faible, le moment magnétique moyen est proportionnel au champ appliqué et inversement proportionnel à la température. II en va de même de l'aimantation : $\boldsymbol{M}_{\boldsymbol{M n}}=\chi_{M n} \boldsymbol{H}$, où la susceptibilité $\chi_{M n}$ est proportionnelle à la densité de spins et inversement proportionnelle à la température (loi de Curie). C'est ce qu'on vérifie expérimentalement sur les semi-conducteurs magnétiques très dilués, par une simple mesure de magnétométrie. Dans des échantillons moins fortement dilués, des interactions antiferromagnétiques viennent diminuer l'aimantation - une complication que nous ignorerons.

Le succès initial des semi-conducteurs magnétiques dilués est dû au fort couplage qui existe entre les spins des porteurs du semi-conducteur et les spins localisés. L'interaction d'échange, entre le spin $s_{e}$ d'un électron de conduction et le spin $S_{M n}$ d'une impureté $\mathbf{M n}$, est proportionnelle au produit scalaire $\boldsymbol{s}_{e} \cdot \boldsymbol{s}_{M r}$. Mais il faut considérer l'effet, sur l'électron de conduction, de toutes les impuretés $\mathrm{Mn}$ réparties aléatoirement sur le réseau cristallin : c'est un problème compliqué, dont il existe une approximation simple, l'approximation dite de « champ moyen ». Elle consiste à remplacer la somme sur tous les opérateurs de spin $\boldsymbol{S}_{M n}$ par la somme de leurs valeurs moyennes, c'est-à-dire, à un facteur près, l'aimantation $\boldsymbol{M}_{\boldsymbol{M n}}$. L'interaction avec les spins $M n$ est alors semblable à un effet Zeeman dans un champ d'échange $\boldsymbol{H}_{\boldsymbol{x}}=\boldsymbol{\lambda} \boldsymbol{M}_{\boldsymbol{M n}}$, qui est proportionnel à l'aimantation et qui s'ajoute au champ appliqué La comparaison d'une mesure de spectroscopie [encadré 1] sur le semi-conducteur hôte d'une part, sur le semi-conducteur magnétique dilué d'autre part, permet de comparer l'effet Zeeman direct et ce nouvel effet qui est qualifié d'effet Zeeman géant.

Cette comparaison permet en effet de mesurer le champ d'échange $\boldsymbol{H}_{x}$, de vérifier qu'il est bien proportionnel à l'aimantation $\boldsymbol{M}_{\boldsymbol{M n}}$ mesurée indépendamment, d'en déduire le paramètre $\lambda$, mais aussi de se rendre compte qu'à basse température, le champ d'échange est plusieurs centaines de fois plus grand que le champ appliqué. Ainsi, à $2 \mathrm{~K}$, pour que l'effet Zeeman soit de l'ordre de grandeur de l'énergie thermique, il faut appliquer un champ de 1 tesla sur $\mathrm{CdTe}$, et de quelques mT seulement sur $\mathrm{Cd}_{0,96} \mathrm{Mn}_{0,04} \mathrm{Te}$ (figure 2).

Ces valeurs ont fait rêver les spécialistes de la magnétooptique : on pourrait envisager une multitude de composants optiques pour moduler la polarisation, l'intensité ou la longueur d'onde d'un faisceau laser. Malheureusement, on a vu que la susceptibilité paramagnétique varie comme l'inverse de la température : l'effet Zeeman géant suit la même évolution, et à température ambiante, il est devenu bien petit ! Néanmoins, ce qu'il en reste suffit pour que des isolateurs optiques à base de $(\mathrm{Cd}, \mathrm{Mn}) \mathrm{Te}$ aient été commercialisés : ces composants évitent que des réflexions parasites renvoient dans la partie active d'un laser une partie du faisceau émis, ce

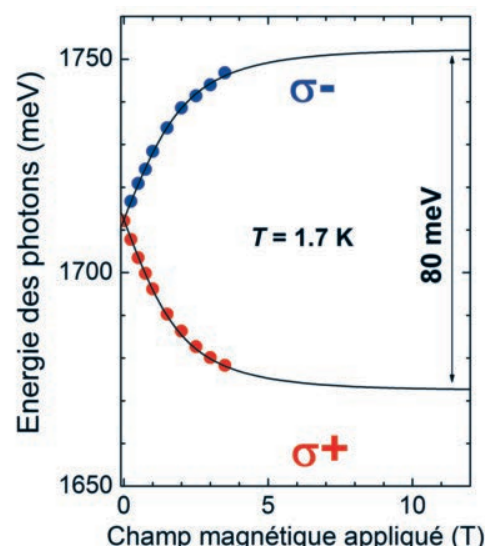

qui perturberait son fonctionnement. Pour cela, ils utilisent une propriété associée à l'effet Zeeman, appelée rotation de Faraday (rotation de la direction de polarisation du faisceau laser transmis). Les composants commercialisés utilisent du (Cd, Mn)Te massif, mais nous avons observé des effets significatifs à température ambiante dans des multicouches de quelques microns. Évidemment, la maîtrise d'un comportement ferromagnétique à température ambiante relancerait l'intérêt des

semi-conducteurs magnétiques dilués pour

des applications en rimentales : lignes : fonctions de Brilloul reproduisant l'aimantation des spins $\mathrm{Mn}$ ). optique, et cela s'ajoute à leur intérêt pour l'électronique de spin.

\section{Semi-conducteurs magnétiques dilués dopés : contrôler le ferromagnétisme}

Le dopage électrique d'un semi-conducteur (non magnétique) crée un système de porteurs libres (électrons occupant le bas de la bande de conduction ou trous occupant le haut de la bande de valence), qui portent un spin 1/2 : si l'on applique un champ magnétique $\boldsymbol{H}$, les deux populations de spin sont différentes et le déséquilibre fait apparaître une aimantation des porteurs $\boldsymbol{M}_{p}=\chi_{\mathrm{p}} \boldsymbol{H}$. La susceptibilité paramagnétique des bandes électroniques (dite de Pauli) $\chi_{p}$ est déterminée par la densité d'états au niveau de Fermi : elle dépend peu de la température, et dans un semi-conducteur massif elle augmente avec le dopage électrique.

Si l'on dope électriquement un semi-conducteur magnétique dilué, on doit tenir compte de l'effet des spins localisés sur les porteurs, caractérisé par le champ d'échange $\boldsymbol{H}_{\boldsymbol{x}}$ comme décrit au paragraphe précédent. Cet effet Zeeman géant s'ajoute à l'effet Zeeman normal, et le domine largement, et on a donc $\boldsymbol{M}_{\boldsymbol{p}}=\chi_{\mathrm{p}}\left(\boldsymbol{H}+\lambda \boldsymbol{M}_{\boldsymbol{M n}}\right) \approx \lambda \boldsymbol{M}_{\boldsymbol{M n}}$. De façon symétrique, les porteurs créent un champ d'échange $\lambda \boldsymbol{M}_{p}$ qui agit sur les spins localisés, et : $\boldsymbol{M}_{\boldsymbol{M n}}=\chi_{\mathrm{Mn}}\left(\boldsymbol{H}+\lambda \boldsymbol{M}_{p}\right)$. En combinant les deux, $\boldsymbol{M}_{M n}=\chi_{M n} \boldsymbol{H} /\left(1-\chi_{p} \lambda^{2} \chi_{M n}\right)$. À basse température, $\chi_{M n}$ devient grand, le dénominateur s'annule, la susceptibilite diverge... le système couplé est devenu ferromagnétique!

Ce modèle très simplifié néglige les effets du désordre lié au caractère aléatoire de la substitution par des ions magnétiques, à la distribution des porteurs et au dopage électrique. II suffit cependant pour comprendre pourquoi une couche de $(\mathrm{Zn}, \mathrm{Mn}) \mathrm{Te}$, habituellement paramagnétique (voire antiferro-

\section{Spectroscopie magnéto-optique}

Le processus de luminescence dans un semi-conducteur correspond à l'émission d'un photon lorsqu'un électron dans la bande de conduction redescend sur un état vide (présence d'un trou) de la bande de valence. On parle de recombinaison électron-trou. L'énergie $h \nu$ du photon est égale à la différence d'énergie entre les deux niveaux. En présence d'un effet Zeeman des bandes (normal ou géant), la raie de luminescence se sépare en deux composantes, polarisées circulairement $\sigma^{+}$et $\sigma^{-}$, pour lesquelles le couplage spin-orbite établit des règles de sélection précises vis-à-vis du spin des porteurs : chaque raie de luminescence teste une composante de spin et l'écart entre les 2 raies traduit les décompositions Zeeman des 2 bandes. La confrontation des spectres magnéto-optiques et des mesures d'aimantation permet d'évaluer précisément les paramètres de couplage caractérisant l'effet Zeeman géant dans chaque bande pour les différents semi-conducteurs magnétiques dilués ceci fait en retour de la spectroscopie magnéto-optique une mesure locale et très sensible de l'aimantation des spins Mn. 
magnétique à cause des interactions directes entre $\mathrm{Mn}$ ) devient ferromagnétique lorsqu'on la dope de type $p$ [2] : la susceptibilité mesurée dans la phase paramagnétique diverge à température finie (figure 3a), et en dessous de cette température une hystérésis apparaît sur les cycles d'aimantation (figure 3b). II faut noter que cette hystérésis apparaît également sur des propriétés des porteurs du semi-conducteur (l'effet Hall, voir figure 3c), ce qui appuie l'idée que le caractère ferromagnétique est une propriété du système couplé spins + porteurs. Des propriétés analogues sont obtenues à plus haute température dans (Ga, Mn)As : jusqu'à $170 \mathrm{~K}$ pour $6 \% \mathrm{Mn}$ [3]. Dans ce cas, l'impureté Mn joue un double rôle, celui de porteur de spin localisé, mais aussi celui de dopant électrique de type $p$ (accepteur).

Un système intéressant est celui d'un puits quantique [encadré 2] $\mathrm{Cd}_{0,96} \mathrm{Mn}_{0,04} \mathrm{Te}$, dans lequel on crée un gaz de trous bidimensionnel en dopant le matériau barrière $\left(\mathrm{Cd}_{0,7} \mathrm{Mg}_{0,3}\right) \mathrm{Te}$. Dans ce cas, la mesure de l'aimantation locale dans le puits est obtenue par spectroscopie optique : une aimantation spontanée induit un effet Zeeman géant (et donc une décomposition de la raie de luminescence en deux raies, voir figure 4) en l'absence de champ appliqué [4].

Le puits quantique est inséré dans une structure à effet de champ, qui permet d'appliquer un champ électrique entre les dopants et le puits. Suivant sa valeur, ce champ maintient les porteurs sur les dopants, ou les amène dans le puits quantique. Dans le premier cas, le système de spins localisés reste paramagnétique et un effet Zeeman n'est observé qu'en présence d'un champ magnétique appliqué. Dans le second cas, les porteurs induisent une interaction ferromagnétique entre les spins $\mathrm{Mn}$, et une aimantation spontanée apparaît en dessous de la température critique $(\approx 3 \mathrm{~K})$. Le champ électrique, obtenu par une tension de grille de l'ordre du volt, permet donc de passer de façon réversible d'un système paramagnétique à un système ferromagnétique : on contrôle les propriétés magnétiques par les techniques de la microélectronique (figure 4). Ajoutons que le même type d'effet est obtenu optiquement, en créant des paires électron-trou dans le matériau barrière : suivant la configuration de champ électrique autour du puits (il y a toujours des transferts de charges dans ces hétérostructures, et donc des champs électriques !), on attire vers le puits préférentiellement les électrons (la densité de trous dans le puits diminue alors) ou les trous (et la densité dans le puits augmente). Ce changement de densité de

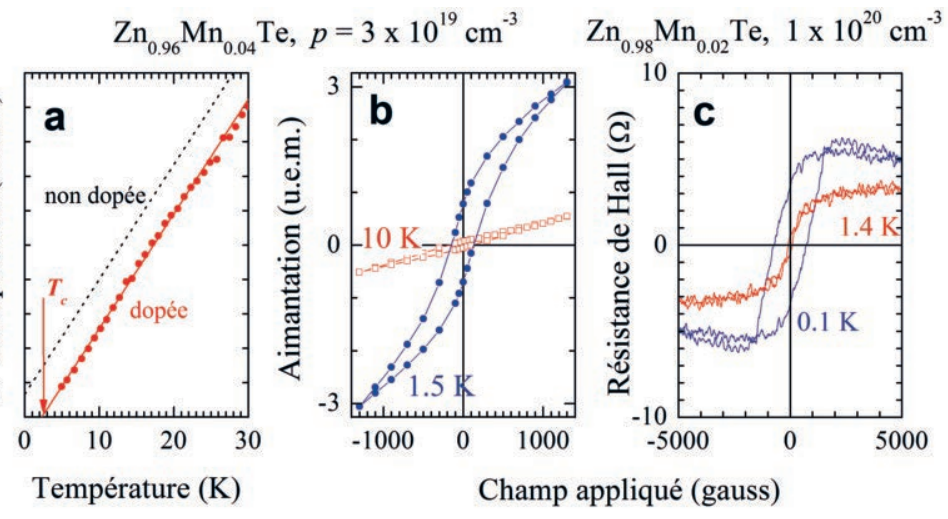

Figure 3. (a) Inverse de la susceptibilité, en fonction de la température, (b) cycles d'aimantation, à deux températures de part et d'autre de la température critique $T_{c} \approx 2,5 \mathrm{~K}$, et (c) effet Hall en fonction du champ magnétique appliqué, à deux températures de part t'autre de la température critique $T_{c} \approx 1 \mathrm{~K}$, pour des couches $(Z n, M n) T e$ dopées de ype $p$ [2].

porteurs peut piloter la transition ferromagnétique, et on peut ainsi espérer inscrire une image dans le système.

\section{Un spin dans une boîte quantique}

Les propriétés décrites jusqu'ici sont des propriétés moyennes, qui concernent un grand nombre d'impuretés magnétiques. Dans le contexte de l'information quantique, on souhaite isoler un spin unique, bien identifié et adressable : c'est ce que permet la spectroscopie de boîtes quantiques individuelles contenant une seule impureté magnétique.

On sait depuis quelques années réaliser la spectroscopie optique de boîtes quantiques individuelles. Pour cela, on élabore un échantillon contenant un plan de boîtes quantiques [encadré 3], de densité relativement faible (quelques $10^{9}$ boîtes par $\mathrm{cm}^{2}$ ) et on sélectionne une zone restreinte de l'échantillon de quelques centaines de nanomètres de côté. Les techniques de lithographie de la micro-électronique rendent cette opération presque facile : on sait ôter le reste de l'échantillon par gravure, ou déposer sur l'échantillon un masque avec un trou. $\mathrm{Si}$ la boîte ne contient pas d'impuretés magnétiques, le spectre de luminescence issu des quelques boîtes ainsi isolées est constitué de raies très fines, dont la position (énergie du photon émis) dépend de la taille de la boîte. En sélectionnant une de ces raies, on sélectionne la boîte correspondante et on réalise ainsi un adressage de cette boîte.

\section{Puits quantique et dopage sélectif}

Le puits quantique est un concept de base des livres de mécanique quantique. II s'agit d'un potentiel de confinement, à l'échelle de quelques nanomètres, qui modifie l'état d'un porteur : celui-ci est caractérisé par des niveaux d'énergie discrets, résultant de la formation d'ondes stationnaires entre les deux interfaces avec les barrières. Une bonne réalisation de cette situation est obtenue en insérant une couche fine d'un semi-conducteur dans un matériau (semi-conducteur ou isolant) où l'énergie minimale des électrons (le bas de la bande de conduction) est plus élevée. Pour éviter que des défauts du matériau perturbent les états électroniques, on veille à ce que le réseau cristallin soit continu à travers l'interface (épitaxie). II existe néanmoins une différence importante : dans le système réel, l'électron est confiné pour ce qui concerne son mouvement dans la direction perpendiculaire à la couche, mais il reste libre de se mouvoir dans le plan de la couche. À ces deux degrés de liberté est associée une énergie cinétique, qui s'ajoute à l'énergie calculée dans les livres de mécanique quantique. Ces énergies marquent donc le minimum d'un continuum (« sous-bande ») et non pas des niveaux discrets. C'est sur ces sous-bandes que se fait la distribution des électrons, en suivant la statistique de Fermi-Dirac. Et c'est entre ces sous-bandes de conduction et des sous-bandes équivalentes pour les trous de la bande de valence, que prend place la photoluminescence.

La meilleure façon d'introduire des électrons libres dans un puits quantique est de doper la barrière adjacente. Dans le matériau massif, il faut fournir une énergie (typiquement, la dizaine ou quelques dizaines de meV, fournie par la température) pour que les électrons quittent le donneur et passent dans la bande de conduction. Dans le cas du dopage sélectif, le niveau d'énergie dans le puits quantique est plus bas, et il y a donc transfert vers le puits, même à basse température. Ce transfert crée un gaz d'électrons bi-dimensionnel. Entre ce gaz d'électrons qui constitue une charge négative, et les donneurs ionisés de charge positive, s'établit un champ électrique. C'est ce champ « de charge d'espace » qui limite le transfert des électrons, et fixe donc la densité (nombre d'électrons par $\mathrm{cm}^{2}$ ) du gaz d'électrons. Les valeurs typiques sont de l'ordre de quelques $10^{11} \mathrm{~cm}^{-2}$. II suffit alors d'ajouter à ce champ de charge d'espace un champ électrique créé en appliquant une tension à l'aide d'un contact métallique « de grille », pour moduler la densité du gaz d'électrons. 


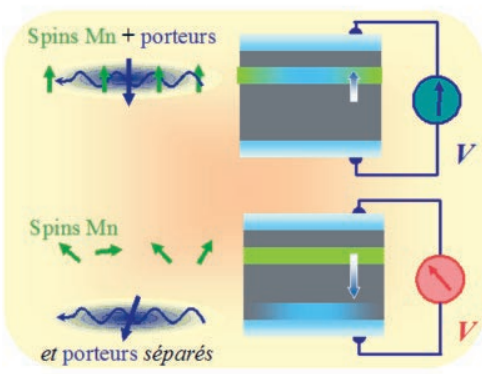

contrôle réversible de la transition para - ferromagnétique par un champ électrique (sans courant)
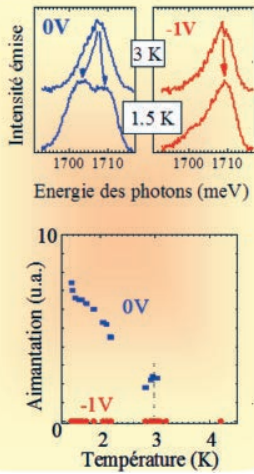

Figure 4. Contrôle de la transition ferromagnétique-paramagnétique par l'application d'un champ électrique. (a) Principe de l'échantillon : le champ électrique créé entre les contacts superpose les porteurs et des spins dans le puits (en haut) ou les sépare (en bas). Lorsque le puits contient les porteurs, le dédoublement de la raie de photoluminescence à basse température traduit l'apparition d'une aimantation spontanée (spectres et données en bleu à droite, $T \approx 3 \mathrm{~K}$ ). Lorsque les porteurs sont séparés, aucune aimantation n'apparaît en l'absence de champ magnétique appliqué, et la raie reste unique à basse température (en rouge) [4].

Si la boîte est fabriquée avec un semi-conducteur magnétique moyennement dilué, comme $\mathrm{Cd}_{0,95} \mathrm{Mn}_{0,05} \mathrm{Te}$, elle contient plusieurs dizaines ou centaines d'ions $\mathrm{Mn}^{2+}$. Une paire électron-trou créée dans la boîte interagit avec ces impuretés magnétiques - plus ou moins, suivant leur position - et le résultat de cette interaction détermine l'énergie exacte du photon émis. Le spectre de luminescence enregistré sur un nombre suffisant d'événements reflète les fluctuations des spins localisés, et la raie est fortement élargie.

La situation est différente si la boîte contient une seule impureté, ce qui est possible si on a ajusté la densité d'impuretés à la densité de boîtes. En présence de la paire électrontrou, on a un objet complexe formé d'un spin $5 / 2$ (celui de l'ion $\mathrm{Mn}^{2+}$ ), d'un spin 1/2 (celui de l'électron), et du spin du trou [5]. L'interaction du spin de l'ion $\mathrm{Mn}^{2+}$ avec la paire électron-trou (qui donnera lieu à l'émission d'un photon d'hélicité donnée) est équivalente à l'effet d'un champ magnétique dirigé perpendiculairement au plan de l'échantillon, qui vaut 1,5 tesla pour une impureté au centre de la boîte. Le moment magnétique de l'ion $\mathrm{Mn}^{2+}$ s'oriente sous l'effet de ce champ, il peut prendre $2 \mathrm{~S}+1=6$ orientations différentes. À chaque orientation correspond une énergie du photon émis, et donc une raie d'émission dans le spectre de luminescence (figure 5). L'émission d'un photon d'énergie et d'hélicité données permet donc de détecter chaque état d'aimantation de l'ion manganèse. Ceci pourrait être un nouveau mode de lecture de mémoires magnétiques pour la « spintronique » du futur.

\section{Perspectives}

Les études actuelles sur les semi-conducteurs magnétiques portent plutôt sur l'arséniure de gallium, et sur la fabrication de nanostructures réalisant certaines fonctions requises par l'électronique de spin en exploitant la polarisa-

tion de spin des porteurs. L'effort se porte aussi sur l'obtention de nouveaux matériaux semi-conducteurs pouvant présenter des propriétés ferromagnétiques à plus haute température, ou offrant une meilleure compatibilité avec le silicium de la microélectronique. Dans tous les cas, les effets de désordre sont plus grands que dans le système modèle que constitue un puits quantique II-VI : aller au-delà du modèle de champ moyen semble une étape incontournable.

L'observation d'un spin unique dans une boîte quantique individuelle ouvre des perspectives très larges. Un spin Mn bien isolé de ses voisins a un temps de relaxation long de plusieurs millisecondes. On pourra lui appliquer une technique déjà utilisée dans $(\mathrm{Cd}, \mathrm{Mn}) \mathrm{Te}$, où les porteurs créés par une impulsion laser de polarisation et de durée bien choisies permettent de manipuler les spins Mn par précession autour du champ d'échange. On peut envisager une spectroscopie électronique des boîtes quantiques, par effet tunnel, déjà réalisée dans d'autres semi-conducteurs, avec une obligation à court terme de mieux contrôler l'élaboration de ces boîtes à un spin : dans l'idéal, chaque boîte contiendrait une impureté magnétique, et une seule, bien positionnée au centre...

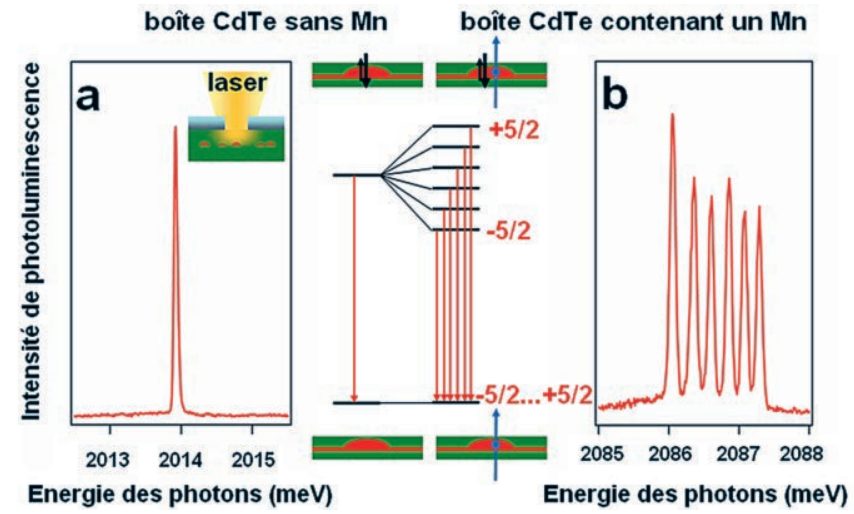

Figure 5. Spectroscopie localisée de la photoluminescence : spectres pou une boîte sans impureté magnétique (a) et avec une seule impureté magnétique (b). Les schémas de niveaux sont donnés au centre de la figure, et le principe de la mesure en insert (en vert, matériau barrière ZnTe, en rouge, CdTe avec ou sans Mn, en bleu, masque d'aluminium) ; les flèches symbolisent les spins des électrons et des trous (en noir), et le spin de l'impureté magnétique (en bleu) [5].

\section{Références}

[1] J. Gaj, R. Planel et G. Fishman, Solid State Commun. 29 (1979) 435

[2] D. Ferrand, J. Cobert, A. Wasiela, C. Bourgognon, S. Tatarenko G. Fishman, T. Andrearczyk, J. Jaroszynski, S. Kolesnik, T. Dietl, B. Barbara et D. Dufeu, Phys. Rev. B 63 (2001) 85201.

[3] K.Y. Wang, R.P. Campion, K.W. Edmonds, M. Sawicki, T. Dietl C.T. Foxon et B.L. Gallagher, AIP Conf. Proc. 772 (2005) 333

[4] H. Boukari, P. Kossacki, M. Bertolini, D. Ferrand, J. Cibert, S. Tatarenko, A. Wasiela, J.A. Gaj et T. Dietl, Phys. Rev. Lett. 88 (2002) 207204

[5] L. Besombes, Y. Léger, M. Maingault, D. Ferrand, H. Mariette et J. Cibert, Phys. Rev. Lett. 93 (2004) 207403.

\section{Boîte quantique}

Un puits quantique correspond à un potentiel de confinement dans une seule direction ; une boîte quantique est obtenue en créant le même type de potentiel suivant les trois directions de l'espace. En pratique, une telle boîte quantique est réalisée en épitaxiant sur un semi-conducteur de type « barrière » (comme ZnTe) un semi-conducteur de type « puits » (comme CdTe), de telle sorte qu'il forme à la surface des îlots de quelques monocouches atomiques d'épaisseur (figure 1d). Lorsqu'on dépose du matériau « barrière » par-dessus, le matériau des îlots est entouré de tous côtés, à l'exception d'une " couche de mouillage " assez fine pour ne pas perturber le confinement (figure 1c).

Dans une telle boîte quantique, le confinement dans les trois directions donne naissance à des niveaux discrets. La photoluminescence a lieu entre ces niveaux discrets : l'énergie des photons émis par une seule boîte est donc parfaitement définie, et le spectre de photoluminescence présente des raies fines. Cependant, cette énergie dépend des dimensions de la boîte : le spectre enregistré sur un ensemble d'un grand nombre de boîtes, dont les dimensions fluctuent de boîte à boîte, montre donc des raies larges. 